\title{
Prediction of invasion depth for submucosal differentiated gastric cancer by magnifying endoscopy with narrow-band imaging
}

\author{
HIDEKI KOBARA $^{1}$, HIROHITO MORI ${ }^{1}$, SHINTARO FUJIHARA ${ }^{1}$, MITSUYOSHI KOBAYASHI ${ }^{1}$, \\ NORIKO NISHIYAMA $^{1}$, TAKAKO NOMURA ${ }^{1}$, KIYOHITO KATO ${ }^{1}$, SHINICHI ISHIHARA ${ }^{3}$, \\ TOSHIAKI MORITO ${ }^{4}$, KOICHI MIZOBUCHI ${ }^{4}$, HISAKAZU IWAMA ${ }^{2}$ and TSUTOMU MASAKI ${ }^{1}$ \\ ${ }^{1}$ Department of Gastroenterology and Neurology, ${ }^{2}$ Life Science Research Center, Kagawa Medical \\ University School of Medicine, Kagawa 761-0793; Departments of ${ }^{3}$ Gastroenterology \\ and ${ }^{4}$ Pathology, Kagawa Rosai Hospital, Kagawa 763-8502, Japan
}

Received January 4, 2012; Accepted February 20, 2012

DOI: $10.3892 /$ or.2012.1889

\begin{abstract}
The usefulness of determining gastric cancer invasion depth by magnifying endoscopy with narrow-band imaging (NBI-ME) has not been established. The objective of our study was to retrospectively compare NBI-ME images of differentiated submucosal (SM) 1 cancer with those of SM2 to identify the indicators of invasion depth for SM2 gastric cancer. Fifteen patients with SM1 differentiated gastric cancer and 20 with SM2 removed by endoscopic submucosal resection (ESD) were included. NBI-ME images matching the invasion depth of pathological specimens were examined to define the following three findings as diagnostic indicators of SM2: non-structure, scattery vessels and multi-caliber vessels. The relationship between indicators and invasion depth and between indicator score and invasion depth was examined in 27 patients (SM1/ SM2: 11/16) with depressed-type gastric cancer (D-GC) and in 8 (SM1/SM2: 4/4) with protruding-type gastric cancer (P-GC). Diagnostic accuracy for invasion depth determined by four endoscopists using regular endoscopic images was compared with that determined by the same endoscopists using NBI-ME. In D-GC, all three indicators were significantly more frequent in SM2 than in SM1 $(\mathrm{p}<0.05)$. All D-GC with $\geq 2$ points were SM2, demonstrating a significant difference in score distribution between SM1 and SM2 (p<0.05). In D-GC, diagnostic accuracy by NBI-ME was higher than that by regular endoscopy by all 4 endoscopists $(\mathrm{p}<0.05)$. NBI-ME findings of non-structure, scattery vessels and multi-caliber vessels can possibly serve as indicators of SM2 invasion in differentiated D-GC. Scoring of the three indicators was significant.
\end{abstract}

Correspondence to: Dr Hideki Kobara, Department of Gastroenterology and Neurology, Kagawa Medical University School of Medicine, 1750-1 Ikenobe, Miki, Kita, Kagawa 761-0793, Japan E-mail: kobara@med.kagawa-u.ac.jp

Key words: submucosal differentiated gastric cancer, invasion depth, magnifying endoscopy

\section{Introduction}

In Japan, cases of early gastric cancer associated with a low possibility of lymph node metastasis have been identified through pathological review of accumulated case reports $(1,2)$. In parallel, the development and spread of endoscopic submucosal dissection (ESD) has enabled en block resection with preserved gastric function (3-5). It is, however, still difficult to accurately determine the invasion depth of gastric cancer, with a number of cases requiring additional surgical resection as a result of diagnostic ESD.

According to the Japanese Gastric Cancer Handling Codes, gastric cancer with a vertical invasion depth of $<500 \mu \mathrm{m}$ is defined as SM1 cancer, and differentiated SM1 gastric cancer with a diameter of $\leq 3 \mathrm{~cm}$ is regarded as an extended indication lesion (6). Therefore, determination of invasion depth plays a very important role in determining the treatment strategy for SM2 gastric cancer, for which additional surgical resection is indicated.

The recent development of endoscopic devices has enabled clearer visualization of fine mucosal and vascular structures under magnified view, making magnifying endoscopy with narrow-band imaging (NBI-ME) an innovative diagnostic tool. In particular, a certain consensus has been reached regarding the effectiveness of magnifying endoscopy for determining invasion depth for intrapapillary capillary loop (IPCL) classification of esophageal cancer (7) and pit-pattern classification of colon cancer (8).

Although NBI-ME has also been shown to be useful for qualitative diagnosis and for determining the range and tissue type of gastric cancer, its usefulness for determining gastric cancer invasion depth has not been established. The objective of this study, therefore, was to evaluate the potential of NBI-ME for determining invasion depth in differentiated early gastric cancer.

\section{Patients and methods}

Of 42 patients who underwent ESD and were given a final pathological diagnosis of SM cancer between April 2007 and 
Table I. Patient background.

\begin{tabular}{|c|c|c|c|}
\hline & SM1 & SM2 & P-value \\
\hline No. & 15 & 20 & NS \\
\hline Mean age (years) & $71.5(50-90)$ & $77.5(69-87)^{a}$ & NS \\
\hline Gender $(\mathrm{M} / \mathrm{F})$ & $14 / 1$ & $16 / 4$ & NS \\
\hline Location $(\mathrm{U} / \mathrm{M} / \mathrm{L})$ & $6 / 5 / 4$ & $5 / 9 / 6$ & NS \\
\hline $\begin{array}{l}\text { Maximum mean } \\
\text { size }(\mathrm{mm})\end{array}$ & $14.7 \quad(3-45)$ & $26.9(6-65)^{a}$ & NS \\
\hline $\begin{array}{l}\text { Form (protruding/ } \\
\text { depressed) }\end{array}$ & $4 / 11$ & $4 / 16$ & NS \\
\hline Ul & 1 & 4 & NS \\
\hline $\begin{array}{l}\text { Pathology (tub1/tub2/ } \\
\text { mixed }^{b} \text { ) }\end{array}$ & $8 / 1 / 6$ & $8 / 1 / 11$ & NS \\
\hline
\end{tabular}

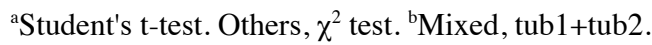

March 2011, 39 patients were evaluable by magnifying endoscopy. A total of 15 patients with SM1 differentiated gastric cancer and 4 with undifferentiated mixed-type SM2 gastric cancer were excluded from this study and the remaining 20 patients with SM2 differentiated gastric cancer were included.

In undifferentiated cancer, the surface of a tumor frequently consists of non-cancerous epithelium. From the histological features, the diagnosis of invasion depth for undifferentiated cancer by NBI-ME that captures only microsurface structure is limited. Therefore, we excluded undifferentiated mixed type of SM2 gastric cancer.

There was no significant difference in patient background factors between the groups ( $p>0.05)$. The maximum mean tumor diameter tended to be larger in SM2 cancer than in SM1 cancer. Morphologically, there was a tendency of a higher proportion of depressed-type cancer in both groups (Table I).

Endoscopic observation was performed using an Olympus GIF-H260Z magnifying endoscope (Tokyo, Japan). The scope was mounted with a tip hood (Elastic Touch M, Top Corp., Tokyo, Japan) to maintain optimal focal distance. Lesions were imaged under regular view, followed by regular magnified view and then by NBI-ME (x60-80). Acetic acid (1.5\% vinegar in tap water) spraying was also used for lesions with obscure fine mucosal structure. A total of $20 \mathrm{ml}$ of the acetic acid solution was injected (about $10 \mathrm{ml}$ at a time) through a forceps channel, with focus adjusted to the region of interest under magnified view. ESD was performed in all cases on the basis of preoperative diagnosis findings. Histopathological diagnosis of resected specimens was performed by a pathologist according to the Gastric Cancer Handling Codes.

NBI-ME images matching the invasion depth of ESD-derived pathological specimens were retrospectively examined to define the following three NBI-ME findings frequently observed in differentiated SM2 cancer as its indicators (Table II).

Typical findings of the indicator 'non-structure' are shown in Fig. 1. Conventional endoscopic images showed a $10-\mathrm{mm}$ red IIc lesion in the lesser curvature of the gastric antrum (Fig. 1A). Mapping images of ESD-resected specimens revealed this lesion to be a well-differentiated adenocarcinoma with mixed invasion depth of M and SM2 (Fig. 1B). Preoperative NBI-ME (x80) of the SM2 invasion site showed an obscure fine mucosal structure (Fig. 1C). Acetic acid spraying enabled clear visualization of glandular ducts and revealed a 'non-structure area' (Fig. 1D).

Typical findings of the indicator 'scattery vessel' are shown in Fig. 2. Conventional endoscopic images showed a $15-\mathrm{mm}$ erosive IIc lesion in the greater curvature of the gastric antrum (Fig. 2A). Mapping images of ESD-resected specimens revealed this lesion to be a well-differentiated adenocarcinoma consisting mainly of M carcinoma with some SM2 invasion (Fig. 2B). Preoperative NBI-ME (x80) of the SM2 invasion site showed two or more scattered blood vessels in an area with obscure fine mucosal structure (Fig. 2C).

Typical findings of the indicator 'multi-caliber vessel' are shown in Fig. 3. Conventional endoscopic images showed a $25-\mathrm{mm}$ red IIc lesion in the anterior wall of the gastric angle in which clipping hemostasis had been performed by a previous surgeon (Fig. 3A). Mapping images of ESD-resected specimens revealed this lesion to be both a well- and moderately-differentiated adenocarcinoma consisting mainly of $\mathrm{M}$ carcinoma with some SM2 invasion (Fig. 3B). Preoperative NBI-ME (x80) of the SM2 invasion site showed an abnormally dilated and tortuous blood vessel with a diameter twice or more that of surrounding tumor vessels (Fig. 3C). We named this abnormal blood vessel a 'multi-caliber vessel'.

Evaluation. In D-GC, the histological architecture of gastric microvilli become lower length in pattern. In contrast, in P-GC, the histological architecture of gastric microvilli become enlonged in pattern. From the histologic differences,

Table II. Definition of indicators for SM2 invasion.

Definition

Indicator 1: loss of fine mucosal structure; 'non-structure'

Indicator 2: scattered blood vessels; 'scattery vessel'

Indicator 3: severely dilated and tortuous blood vessels; 'multi-caliber vessel'
Areas in which no glandular duct is observed, either under magnified view or after acetic acid spraying, with a size corresponding to $\geq 2$ residual glandular ducts (Fig. 1).

Presence of $\geq 2$ scattered blood vessels in an area with obscure mucosal structure (Fig. 2).

Presence of tortuous blood vessels with a diameter twice or more that of surrounding tumor vessels (Fig. 3). 
A
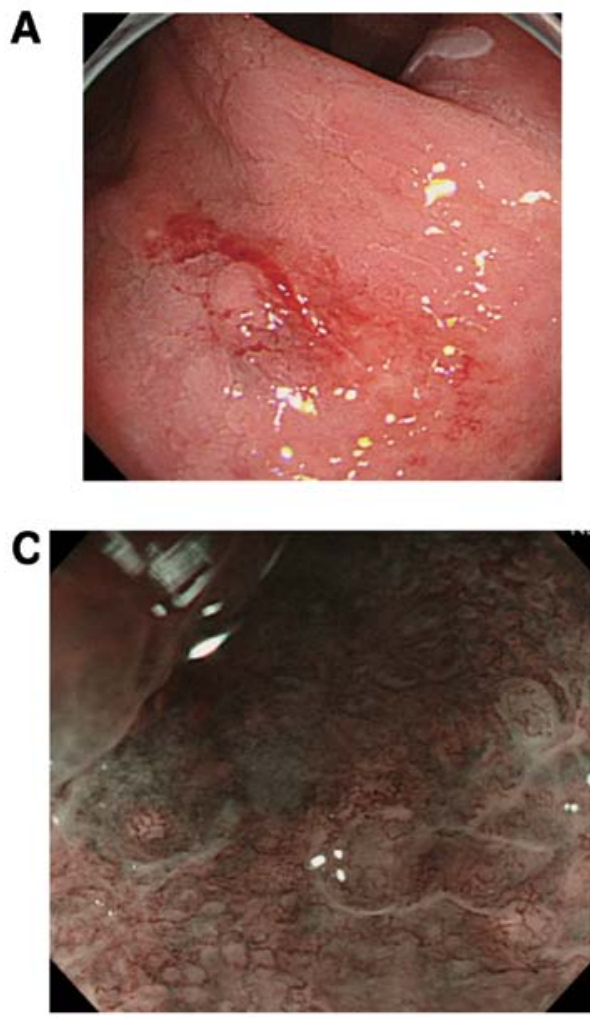

B
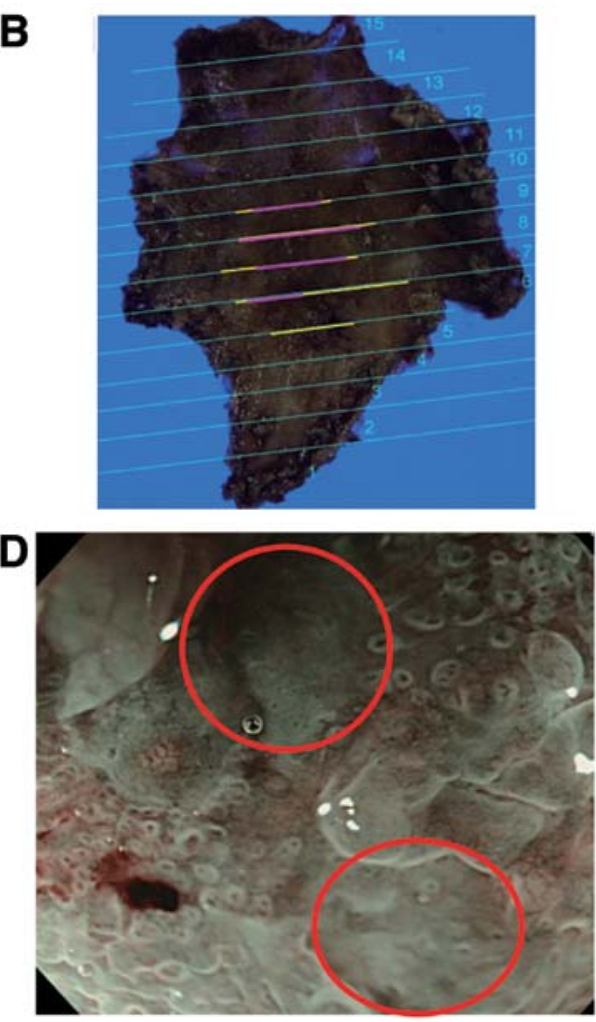

Figure 1. Typical findings of indicator 'non-structure'. (A) A conventional endoscopic image showing a 10-mm red IIc lesion in the lesser curvature of the gastric antrum. (B) A mapping image of ESD-resected specimens showing a well-differentiated adenocarcinoma with mixed invasion depths of M (yellow line) and SM2 (red line). (C) A preoperative NBI magnifying endoscopic image (x80) of the SM2 invasion site showing an obscure fine mucosal structure. (D) Acetic acid spraying enabled clear visualization glandular ducts and revealed a 'non-structure area' (surrounded by a red line).
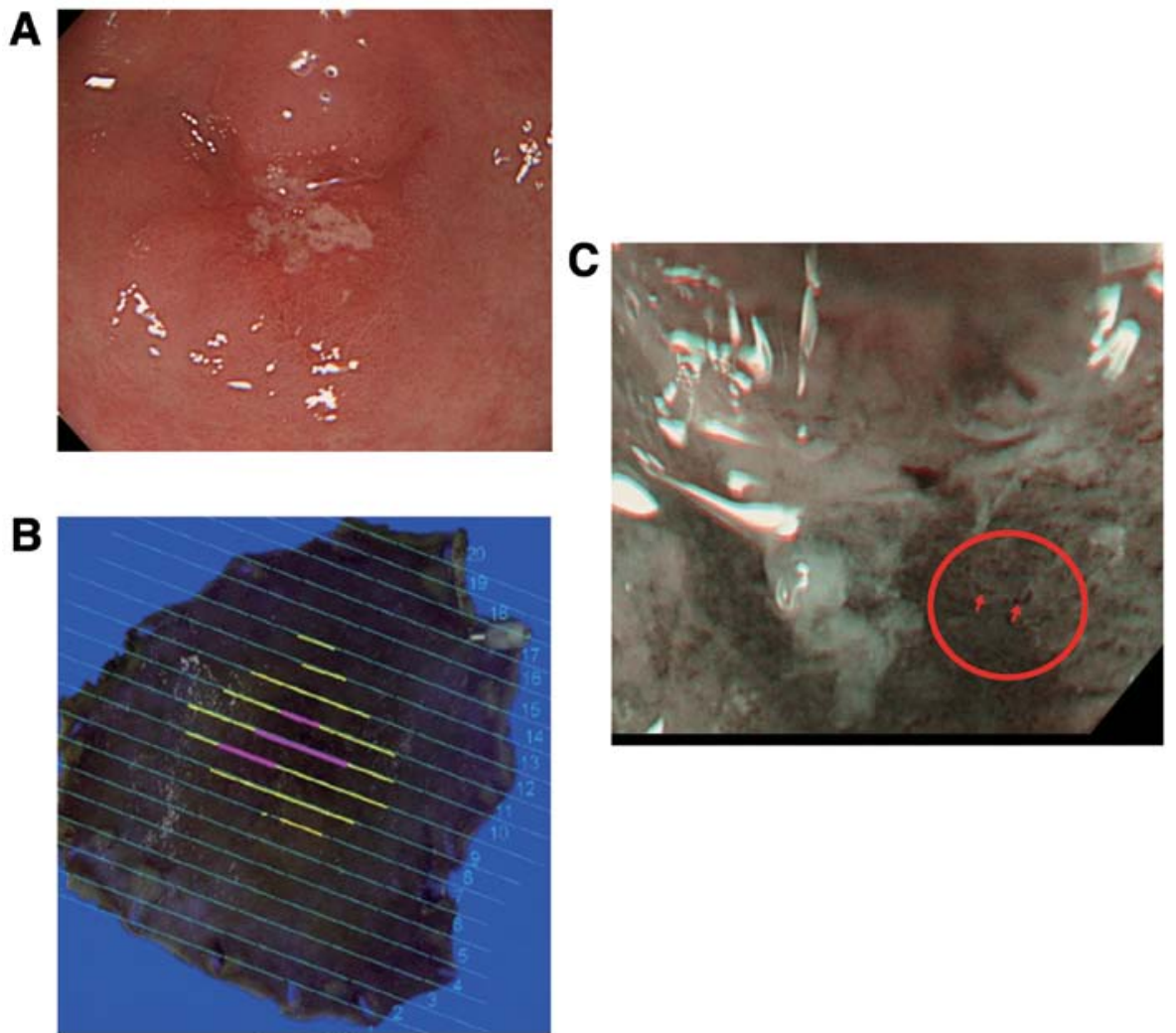

Figure 2. Typical findings of indicator 'scattery vessel'. (A) A conventional endoscopic images showing a 15-mm erosive IIc lesion in the greater curvature of the gastric antrum. (B) A mapping image of ESD-resected specimens showing a well-differentiated adenocarcinoma consisting mainly of M carcinoma (yellow line) with some SM2 invasion (red line). (C) A preoperative NBI magnifying endoscopic image (x60) of the SM2 invasion site showing two or more scattered blood vessels (in the red circle and arrows) in an area with obscure fine mucosal structure. 

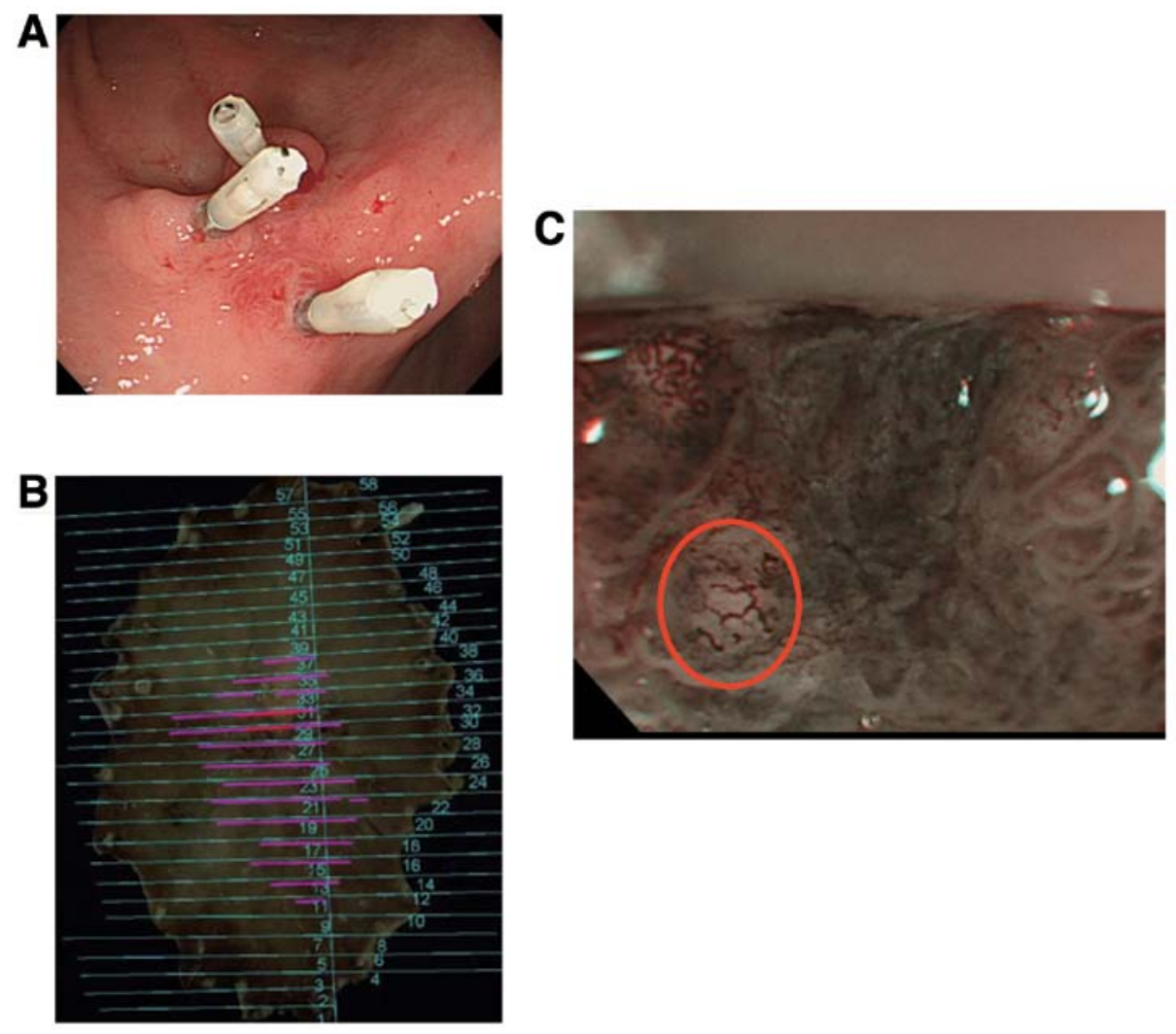

Figure 3. Typical findings of indicator 'multi-caliber vessel'. (A) A conventional endoscopic image showing a 25-mm red IIc lesion in the anterior wall of the gastric angle in which clipping hemostasis had been performed by a previous surgeon. (B) A mapping image of ESD-resected specimens showing a well- and moderately-differentiated adenocarcinoma consisting mainly of M carcinoma (pink line) with some SM2 invasion (red line). (C) A preoperative NBI-ME (x80) of the SM2 invasion site showing an abnormally dilated and tortuous blood vessel (surrounded by a red line) with a diameter twice or more that of surrounding tumor vessels. This abnormal blood vessel was named a 'multi-caliber vessel'.

we examined SM differentiated gastric cancer according to morphology.

Evaluation: assessment I. The following items were evaluated separately in 27 patients with depressed-type gastric cancer (SM1/SM2: 11/16) and in 8 patients with protruding-type cancer (SM1/SM2: 4/4). I-1) The relationship between the three indicators and invasion depth. I-2) The relationship between scores for the three indicators and invasion depth. An endoscopist blinded to regular endoscopic findings and pathological diagnosis calculated scores for each patient according to the presence ( 1 point) or absence ( 0 point) of each indicator, with a maximum possible score of 3 points.

Evaluation: assessment II. The diagnostic accuracy of the determination of invasion depth by regular endoscopy was compared with that by magnifying endoscopy for the following items. II-1) After randomizing SM1 and SM2 cases, four endoscopists blinded to invasion depth were asked to determine this factor using regular endoscopic images, after which diagnostic accuracy was determined for each endoscopist. II-2) As in assessment I, four endoscopists calculated scores according to the presence or absence of indicators in each case. For each endoscopist, diagnostic accuracy was determined by assuming that all patients with a score of $\geq 2$ points ( $\geq 2$ indicators) and showing significant differences, had SM2 cancer.
Statistical analysis. Intergroup comparison of patient background factors was performed using the Student's t-test or $\chi^{2}$ test. The relationships between the three indicators and invasion depth and between scores for the indicators and invasion depth were analyzed using the $\chi^{2}$ test. The Student's t-test was used to compare diagnostic accuracy for invasion depth by regular endoscopy and that by magnifying endoscopy. A p-value of $<0.05$ was considered significant.

\section{Results}

Assessment I-1: relationship between indicators and invasion depth. In patients with depressed-type gastric cancer, indicator 'non-structure' was observed significantly more frequently in SM2 cases $(14 / 16,88 \%)$ than in SM1 cases $(2 / 11,18 \%)$ $(\mathrm{p}=0.002)$. Indicator 'scattery vessel' was also significantly more frequently observed in SM2 cases $(12 / 16,75 \%)$ than in SM1 cases $(0 / 11,0 \%)(\mathrm{p}<0.0001)$. Similarly, indicator 'multicaliber vessel' was significantly more frequently observed in SM2 cases $(7 / 16,44 \%)$ than in SM1 cases $(0 / 11,0 \%)(p=0.01)$. Thus, in patients with depressed-type gastric cancer, all indicators were significantly more frequently observed in SM2 than in SM1 cases (Fig. 4A).

On the other hand, in patients with protruding-type gastric cancer, indicator 'non-structure' was observed in 2 of $4(50 \%)$ SM1 cases and in 3 of 4 (75\%) SM2 cases, with no significant 

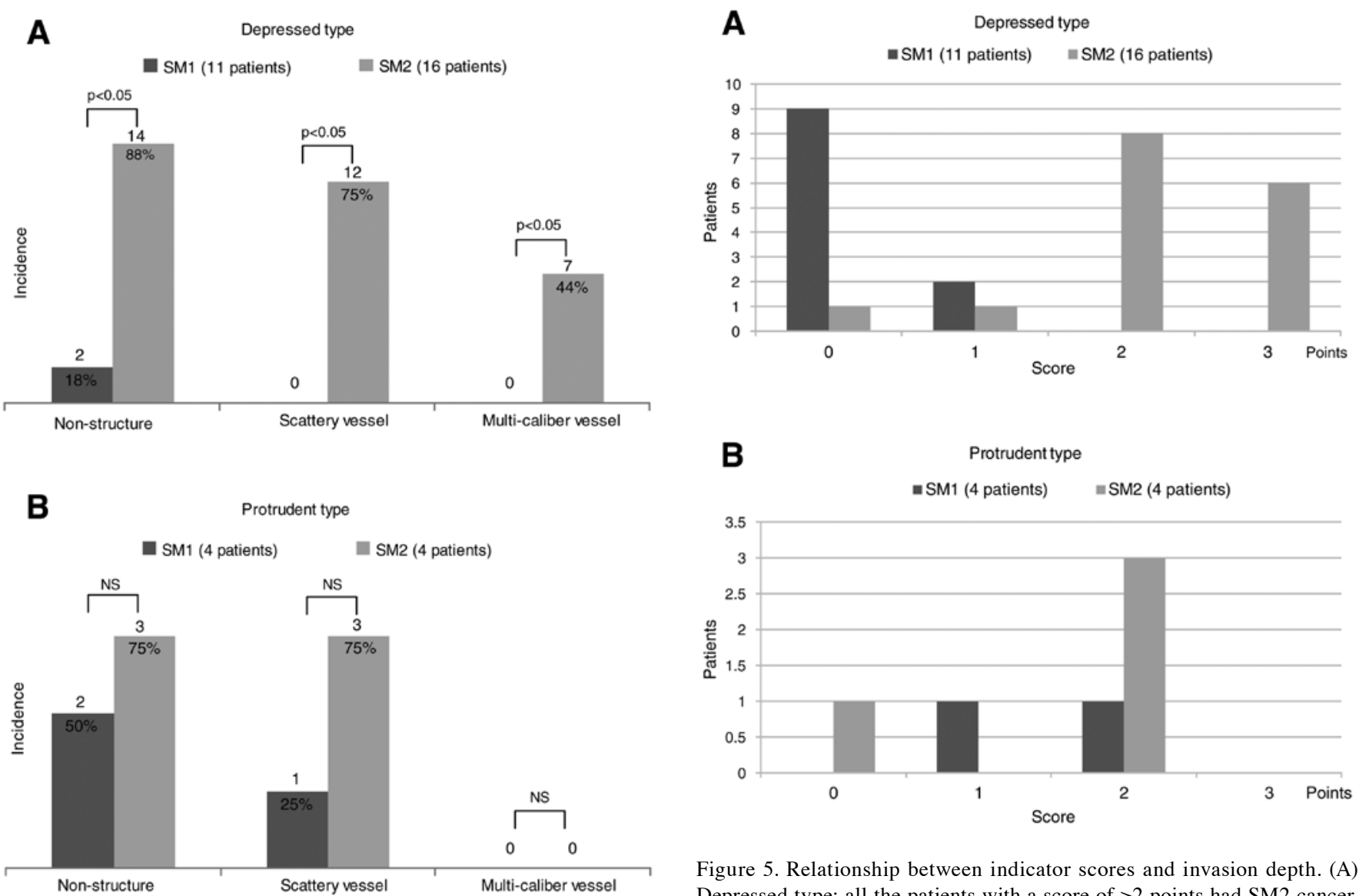

Figure 4. Relationship between indicators and invasion depth. (A) Depressed type: all three indicators were significantly more frequent in SM2 than in SM1 cases $(\mathrm{p}<0.05)$. (B) Protruding type: the frequency of all three indicators was similar between SM2 and SM1 cases ( $>0.05)$.

difference in frequency $(\mathrm{p}=0.465)$. Indicator 'scattery vessel' was observed in 1 of 4 (25\%) SM1 cases and in 3 of 4 (75\%) SM2 cases, again with no significant difference in frequency $(\mathrm{p}=0.157)$. Indicator 'multi-caliber vessel' was not observed in any of the patients in either group. Thus, in patients with protruding-type gastric cancer, no significant difference was observed in the frequency of each indicator between groups (Fig. 4B).

Assessment I-2: relationship between indicator score and invasion depth. Of the 11 patients with SM1 depressed-type gastric cancer, 9 and 2 patients had a score of 0 and 1 point, respectively, with no patients having a score of $\geq 2$ points. Of the 16 patients with SM2 depressed-type cancer, 1, 1, 8 and 6 patients had $0,1,2$ and 3 points, respectively. Of the 8 patients with 2 points, 6 were positive for indicators 'non-structure' and 'scattery vessel' and 2 patients were positive for 'nonstructure' and 'multi-caliber vessel'. Thus, all patients with a score of $\geq 2$ points had SM2 cancer, indicating a significant difference in score distribution between SM1 and SM2 cases $(\mathrm{p}<0.0001)$ (Fig. 5A).

Of patients with protruding-type gastric cancer, 2, 1 and 1 of 4 patients with SM1 cancer had 0, 1 and 2 points, respectively, and 1, 0, 3 and 0 of 4 patients with SM2 cancer had $0,1,2$ and 3 points, respectively. Thus, among patients with

protruding-type gastric cancer, no significant difference was observed in score distribution between SM1 and SM2 cases $(\mathrm{P}>0.05)$ (Fig. 5B).

Assessment II: comparison of diagnostic accuracy for invasion depth by regular endoscopy and that by magnifying endoscopy. For depressed-type gastric cancer, diagnostic accuracy for invasion depth by regular and magnifying endoscopy was 67 and $89 \%$ by endoscopist $A, 70$ and $81 \%$ by endoscopist B, 70 and $78 \%$ by endoscopist $C$ and 59 and $67 \%$ by endoscopist $D$, respectively. Assuming that all patients with a score of $\geq 2$ points had SM2 cancer, diagnostic accuracy by magnifying endoscopy was higher than that by regular endoscopy for all 4 endoscopists, indicating a significantly higher diagnostic accuracy of magnifying endoscopy ( $\mathrm{p}=0.044)$ (Fig. 6A).

For protruding-type gastric cancer, diagnostic accuracy by regular and magnifying endoscopy was 63 and $75 \%$ by endoscopist A, 75 and $75 \%$ by endoscopist B, 50 and $63 \%$ by endoscopist $\mathrm{C}$ and 75 and $63 \%$ by endoscopist $\mathrm{D}$, respectively, with no significant difference in diagnostic accuracy between the two endoscopic procedures ( $\mathrm{p}=0.170$ ) (Fig. 6B).

\section{Discussion}

Accurately assessing invasion depth is very important for determining the treatment strategy for gastric cancer. This is 

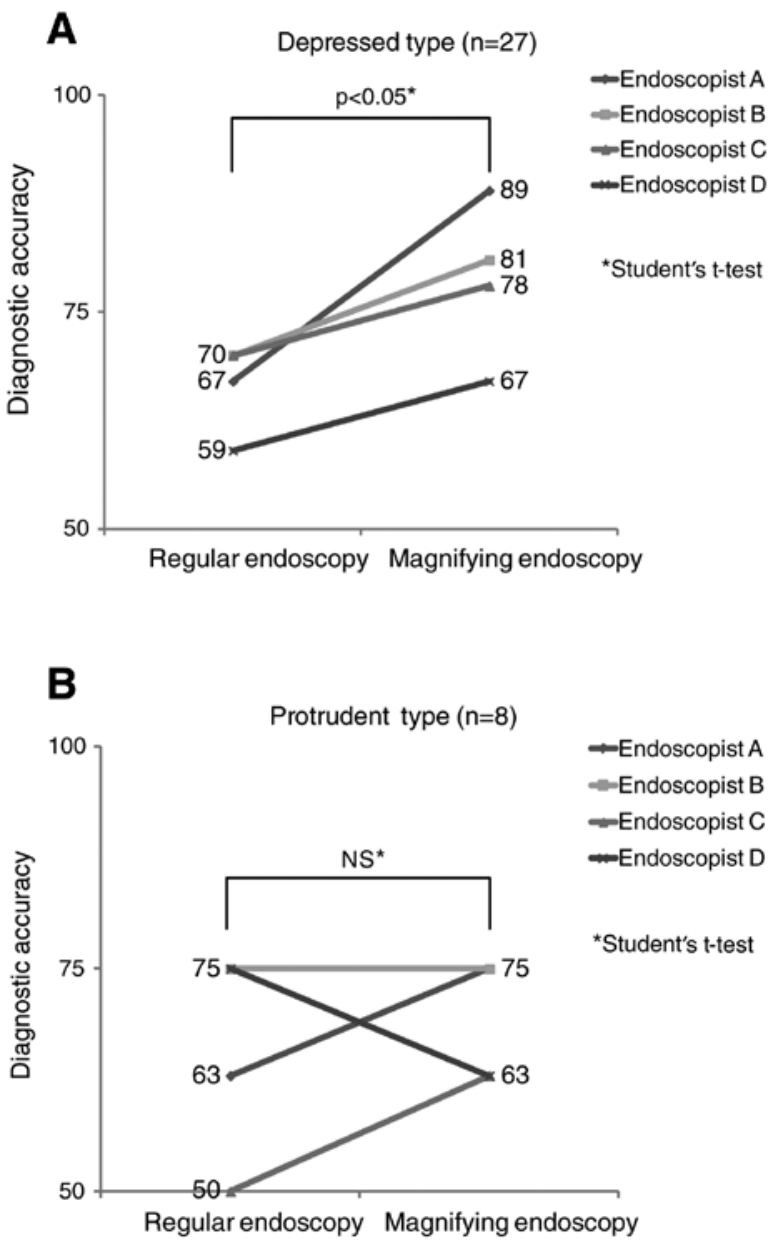

Figure 6. Comparison of diagnostic accuracy for invasion depth by regular endoscopy and by magnifying endoscopy. (A) Depressed type: when assuming that patients with a score of $\geq 2$ points had SM2 cancer, diagnostic accuracy by magnifying endoscopy was higher than that by regular endoscopy by all 4 endoscopists, indicating a significantly higher diagnostic accuracy of magnifying endoscopy $(\mathrm{p}<0.05)$. (B) Protruding type: no significant difference was observed in diagnostic accuracy between the two endoscopic procedures $(\mathrm{p}>0.05)$.

usually done using a combination of regular endoscopy, gastric fluoroscopy and endoscopic ultrasound techniques which provide a certain degree of diagnostic accuracy.

Recently, a consensus has been reached regarding the effectiveness of magnifying endoscopy for determining invasion depth for IPCL classification of esophageal cancer (7) and pitpattern classification of colon cancer (8). Although magnifying endoscopy has been used for observation of gastric mucosa for many years $(9,10)$, modification of gastric mucosa by acid and chronic inflammation caused by Helicobacter pylori infection complicates evaluation of the mucosal fine structure on magnified images and have delayed the development of this technique. The recent development of narrow-band imaging (NBI), a procedure of image-enhanced endoscopic imaging, has improved the visibility of fine mucosal and vascular structures of gastric cancer and is being established as a new diagnostic tool.

Magnifying endoscopy has been shown to be effective for differentiating benign from malignant tumors (11), determining tissue type (12) and determining the extent of gastric cancer (13). Acetic acid spraying is also an effective strategy for determining the extent of cancer in which the cancerous portion is observed as a red area against a white background of normal mucosa. A recent study suggests that the use of acetic acid spraying combined with endoscopy enables detailed observation of surface structures (14). In the present study, acetic acid spraying in patients with obscure fine mucosal structure also enabled confirmation of the presence or absence of normal structure. With a limited number of studies available, no consensus has been reached regarding the usefulness of NBI-ME for determining invasion depth in gastric cancer. A review of preoperative magnified images of SM1 and SM2 invasion sites revealed a higher frequency of 'non-structure', 'scattery vessel' and 'multi-caliber vessel' in differentiated SM2 cancer. We thus evaluated the usefulness of these findings as indicators of SM2 cancer. Results revealed a significant difference in the frequency of the indicators between SM1 and SM2 cases of depressed-type gastric cancer, demonstrating their potential as indicators of SM2 invasion. In assessment II, patients with depressed-type gastric cancer with a score of $\geq 2$ points ( 2 or more indicators) had SM2 cancer significantly more frequently than SM1 cancer, suggesting the usefulness of scoring the indicators.

When assuming that all patients with depressed-type gastric cancer with a score of $\geq 2$ points had SM2 cancer, diagnostic accuracy for invasion depth by magnifying endoscopy was significantly higher than that by regular endoscopy, objectively demonstrating the usefulness of the scoring system itself.

In patients with protruding gastric cancer, indicator 'scattery vessel' was more frequently observed in SM2 cases $(3 / 4,75 \%)$ than in SM1 cases $(1 / 4,25 \%)$. Of the 4 patients with SM2 cancer, 3 patients (75\%) had a score of 2 . Although no statistically significant difference was observed due to a small number of patients, a certain trend was observed in score distribution among patients with protruding-type cancer, as was among those with depressed-type cancer. At the same time, of the 4 patients with SM1 cancer, $2(50 \%)$ patients had indicator 'non-structure', $1(25 \%)$ had indicator 'scattery vessel' and $1(25 \%)$ had a score of 2 points, suggesting that some patients have magnified endoscopic findings that do not correspond to any of the indicators. Regular endoscopic findings of SM deep invasion sites in patients with protruding-type gastric cancer are characterized by a gradual rise appearing like a submucosal tumor, tense feeling and double protrusion as a result of the formation of a cancerous mass in the submucosa. More case reports are needed to verify the usefulness of magnifying endoscopy compared with diagnosis on the basis of characteristic findings by regular endoscopy.

Several investigators have reported that SM cancer is associated with the appearance of non-structure areas in fine mucosal structure, irregularly-shaped, dilated or extended microvessels and evidence of a hypovascular tumor $(15,16)$. A decreased degree of tumor differentiation has been associated with the appearance of open loop, tortuous, and bizarre-type abnormal microvessels (17). The fact that similar magnifying endoscopic findings were obtained in the present study may suggest a certain trend.

Only a limited number of studies, including the present study, have statistically examined the frequency of magnifying 
endoscopic findings between SM1 and SM2 cases. None of the previous studies have scored indicators or compared diagnostic accuracy, as was performed in assessments II and III. Thus, the present study is notable in that it examined the determination of invasion depth in SM2 cancer cases from an unprecedented point of view.

One of the remaining issues is to obtain pathological proof of the histological characteristics of tumors by using the indicators to assess a deep invasion site. Because magnifying endoscopy shows only the structure and vascular architecture of the mucosal surface, histological findings in a deep invasion site can only be estimated from differences in surface structure. We currently consider that the 'non-structure' aspect indirectly reflects a decreased density of glandular ducts and desmoplastic reaction due to deep invasion of cancer. With regard to microvascular changes, indicator 'scattery vessel' appears to represent a decreased vascular density due to an increase or expansion of stromal components associated with deep invasion of cancer. Indicator 'multi-caliber vessel' appears to reflect the presence of severely dilated and tortuous capillaries with various diameters in the stroma between glandular ducts.

Further studies of a prospective design with one-to-one comparisons of magnified endoscopic images with corresponding histological images of deep invasion sites are thus needed to obtain histological evidence supporting the present findings, with special attention paid to the amount of stroma and vascular architecture.

Finally, if the surface of a tumor consists of non-cancerous epithelium, it is not necessarily undifferentiated cancer with an extended middle mucosal layer, but may be a special type of differentiated cancer. This is one of the limitations of magnifying endoscopy as it only observes the mucosal surface; thus, this diagnostic strategy is not necessarily applicable to all kinds of lesions. Nevertheless, the present study has suggested the usefulness of magnifying endoscopy for determining invasion depth in differentiated, depressed-type gastric cancer, the most common type of gastric cancer lesion.

In conclusion, magnifying endoscopic findings of 'nonstructure', 'scattery vessel' and 'multi-caliber vessel' can possibly serve as indicators of SM2 invasion when determining the invasion depth of depressed-type gastric cancer. Patients with depressed-type gastric cancer and a score of $\geq 2$ points ( 2 or more indicators) had SM2 cancer significantly more frequently than SM1 cancer, suggesting the usefulness of scoring the indicators.

\section{References}

1. Gotoda T, Yanagisawa A, Sasako M, et al: Incidence of lymph node metastasis from early gastric cancer: estimation with a large number of cases at two large centers. Gastric Cancer 3: 219-225, 2000.

2. Hanaoka N, Tanabe S, Mikami T, et al: Mixed-histologic-type submucosal invasive gastric cancer as a risk factor for lymph node metastasis: feasibility of endoscopic submucosal dissection. Endoscopy 41: 427-432, 2009.

3. Tada M, Murakami A, Karita M, et al: Endoscopic dissection of early gastric cancer. Endoscopy 25: 445-450, 1993.

4. Yahagi N, Fujishiro M, Kakushima N, et al: Endoscopic submucosal dissection for early gastric cancer using the tip of an electro-surgical snare (thin type). Dig Endosc 16: 34-38, 2004.

5. Ono H, Kondo H, Gotoda T, et al: Endoscopic mucosal dissection for treatment of early gastric cancer. Gut 48: 225-229, 2001.

6. Japanese Gastric Cancer Association: Gastric Cancer Treatment Guidelines. 3rd edition. Kanehara, Tokyo, 2010.

7. Inoue H: Magnification endoscopy in the esophagus and stomach. Digestive Endoscopy 13: S40-S41, 2001.

8. Kudo S, Tamura S, Nakajima T, et al: Diagnosis of colorectal tumorous lesions by magnifying endoscopy. Gastrointest Endosc 44: 8-14, 1996.

9. Salem SN and Truelove SC: Dissecting microscope appearance of the gastric mucosa. Br Med J 2: 1503-1504, 1964.

10. Sakaki N, Iida Y, Okazaki Y, et al: Magnifying endoscopic observation of the gastric mucosa, particularly in patients with atrophic gastritis. Endoscopy 10: 269-274, 1978.

11. Yao K, Oishi T, Matsui T, et al: Novel magnified endoscopic findings of microvascular architecture in intramucosal gastric cancer. Gastrointest Endoscopy 56: 279-284, 2002.

12. Nakayoshi T, Tajiri H, Matsuda K, et al: Magnifying endoscopy combined with narrow band imaging system for early gastric cancer: correlation of vascular pattern with histopathology (including video). Endoscopy 36: 1080-1084, 2004.

13. Nagahama T, Yao K, Maki S, et al: Advantage of magnifying endoscopy with narrow-band imaging (NBI) over standard endoscopy for determining the margins of lateral extent of early gastric cancer. Endoscopy 42: A99, 2010.

14. Oyama T, Tomori A, Ishii E, et al: Histopathological diagnosis of gastric cancers by magnifying endoscopy with NBI. Stomach Intestine 46: 943-955, 2010

15. Otsuka $\mathrm{Y}$, Niwa $\mathrm{Y}$, Ohmiya N, et al: Usefulness of magnifying endoscopy in the diagnosis of early gastric cancer. Endoscopy 36: 165-169, 2004.

16. Ono S, Kato M, Itoh T, et al: Diagnosis of intestinal type gastric cancer with SM1 invasion by magnifying endoscopic findings. Stomach Intestine 42: 99-109, 2007.

17. Takeuchi Y, Ishii H, Yao K, et al: Histopathological diagnosis of gastric cancer using magnifying endoscopy combined with the narrow band imaging - a proposal of classification for morphological findings according to VS classification. Stomach Intestine 46: 943-955, 2011. 